\title{
Agent Reasoning in Negotiation
}

\author{
Katia Sycara and Tinglong Dai
}

\begin{abstract}
Negotiation has been studied in different communities both scientific and communities of practice. The social sciences and the mathematical sciences have investigated different aspects of negotiation with different goals: the goals of the social sciences are to understand the factors and reasoning processes that underlie human negotiation behavior. The goal of the mathematical sciences is to formulate mathematical models that capture elements of negotiation. Further, the mathematical models can be divided into analytic models (economic, operations research etc) and computational models. The aim of the analytic models is to provide guarantees of their behavior, characterizations of optimality, or provide managerial guidance to optimize negotiation activity. The computational models aim to provide computational tractability through approximation algorithms and heuristics. Most crucially, the computational research aims to have the models implemented in autonomous processes, called agents, that are able to incorporate realistic factors of negotiation (e.g. argumentation, information seeking, and cognitive factors) and engage in negotiations in a decentralized manner. Such agent models promise to contribute to our understanding of human information processing in negotiation. Additionally, they could be used for decision support of human decision makers. In the long run, such models can even become substitutes for human negotiators. In this chapter we will provide a selective review of the most important works in the analytic and computational negotiation literature, point out some differences and synergies and provide pointers to open questions and future research.
\end{abstract}

Katia Sycara

Robotics Institute, Carnegie Mellon University, 5000 Forbes Avenue, Pittsburgh, PA 15213, USA. e-mail: katia@cs.cmu.edu

Tinglong Dai

Tepper School of Business, Carnegie Mellon University, 5000 Forbes Avenue, Pittsburgh, PA 15213, USA. e-mail: dai@cmu.edu 


\section{Introduction}

Negotiation is a process among self-interested agents with the purpose of reaching an agreement that satisfies preferences and constraints of the concerned parties. As a process, negotiation has the following characteristics: (a) it is decentralized, (b) it involves communication among the parties, (c) it involves incomplete information (e.g. the utilities of the parties are private knowledge to each party), (d) it encompasses possibly conflicting preferences over actions and outcomes. Additionally, the process of negotiation (except in its most simplified form) is not well structured, in the sense that there are no well defined rules for creating legal sequences of communication actions. For example, an offer by party A may be followed by party B's request for information to further clarify conditions of the offer, or by an argument to convey to A that the offer is not fair, or by a rejection, or by a counter-offer. These characteristics of negotiation make it quite distinct from other forms of selfinterested interactions, especially auctions. In auctions, the process is not totally decentralized but it requires the presence of a centralized auctioneer, the parties do not communicate directly, and the interaction protocol is well specified ${ }^{1}$.

Negotiation is a very common process in human affairs and the negotiation literature is vast encompassing research within Economics (e.g., (Kim 1996), (Conlin and Furusawa 2000)), Political Science (e.g.,(Carnevale and Lawler 1986), (Licklider 1995)), Sociology (e.g.,(Rhoades and Slaughter 1991), (Netto 2008)), Psychology (e.g., (Thompson 1991), (Morre 2002)), Organizational Behavior (e.g., (Lewicki, Weiss, and Lewin 1992), (Dreu 2003)), Decision Sciences (e.g., (Sebenius 1992)), Operations Research (e.g., (Ulvila and Snider 1980), (Fogelman-Soulie, Munier, and Shakun 1983) and (Muthoo 1995)), Mathematics (e.g., (Froncek 2009)), and, most recently, Computer Science (see (Kraus 1997) for a review of literature until 1997). In general, the goal of investigating negotiation in the social sciences is to understand the factors involved in negotiation among people, whereas the goal of the economics and mathematical sciences is to provide analytical formalizations of negotiation so that decision making processes that lead to optimal negotiation outcomes could be discovered, and advice to decision makers could be provided as to how to implement and utilize these formulations in practice. In this respect, the aim of the social science negotiation research (See Chapters 4, 6, and 7 of this volume for example) is descriptive whereas the aim of the mathematical science research is prescriptive.

Within the mathematical sciences camp we differentiate goals and approaches of economics and operations research (called thereafter for simplicity analytic approaches) on the one hand and computer science on the other (computational approaches). The analytic approaches, dominated by the game theoretic view point, have focused primarily on producing models that could be mathematically characterized and solved. Typically, the computational complexity of algorithms for achieving a solution has not been the main focus; neither have concerns for sim-

${ }^{1}$ Many authors, e.g., (Bichler 2000), refer to auctions as a form of negotiation, which results in confusion in terminology. 
ilarity of the analytical models to human reasoning. A notable exception has been work by behavioral economists and decision scientists that have challenged game theoretic assumptions (see (Rothkopf 1983), (Roth 1985) and (Neale and Bazerman 1985 ) for example) and have produced models that are based on bounded rationality. Additionally, due to the desire to characterize ways to achieve optimal outcomes, analytic models simplify the negotiation process to sequences of offers and counter offers and focus on how optimal outcomes could be produced. On the other hand, the focus of the computational literature has been on (a) computationally characterizing the complexity of negotiation (b) finding computationally tractable algorithms, (c) creating computational agents that embody reasoning that includes cognitive considerations. Moreover, the computational models are aimed at being utilized in different theoretical and practical endeavors.

A distinction that has not received much attention in the literature is one of centralized vs. decentralized computation. The solution finding procedures in the analytic models is centralized. This necessitates various fictitious devices, such as simulation of the game in game theory, the submission of simultaneous offers, and the invention of signaling. In other words, the calculation of the equilibria is done in a centralized way and the execution is envisioned to be decentralized. Many computational models, as well, are using centralized algorithms. However, one of the challenges is to embody the algorithms in separate autonomous computational agents that calculate the next step in the negotiation after observing the previous step. This poses interesting theoretical and computational issues since (a) the autonomous calculation is on line and thus must be efficient, and (b) in multiparty interactions, there is an additional issue of how the order of interaction of the agents is determined.

The analytic and computational approaches are synergistic. Analytic models provide certain guarantees of the solution concepts although by necessity cannot encompass the complexities of real negotiations or consider contextual or cognitive factors. The computational models, on the other hand, relying on approximate algorithms and heuristics have the flexibility to include cognitive considerations and features of human reasoning, thereby promising to contribute to our understanding of human information processing in negotiation. Additionally, such models could be used for decision support of human decision makers, either as trusted third parties (mediators) or directly supporting their owner. In the long run, such models can even become substitutes for human mediators or negotiators.

Mathematical models are currently oversimplified versions of reality. They generally make the assumption that the negotiation process is well structured where negotiation actions occur and result in agreement or opting out of the negotiation. In real situations, however, the parties may take actions to change the structure of the negotiation itself, for example adding or subtracting issues as the environment changes or as the parties try to enlarge the pie (cf., (Shakun 1991), (Kersten et al. 1991) and (Sebenius 1992)). Research is very far from being able to model or derive automated ways to do such restructuring, but some initial attempts have been made (Sycara 1991). The basic elements of negotiation are the underlying interests and social motives of the participants, and their interactions, e.g. creating value or claiming value, which respectively characterize integrative vs. distributive negotia- 
tions. A critical area of interaction is persuasion, i.e. how one party can convince the other to accept a particular proposal or resolve some impasse. Various types of arguments and justifications can be offered to this end. Finally, these interactions become operationalized through observable communication actions, such as making proposals, counterproposals, asking for clarification, asking for the preferences of another party etc. These observable actions along with an understanding of what activity sequences are coherent constitute the protocol of negotiation. What particular linguistic expressions to use during each of the communication actions in negotiation has been an area of considerable research (e.g., (Lambert and Carberry 1992) and (Lochbaum 1998)) but it is outside of the scope of the current chapter.

In this chapter we will focus on work in the mathematical sciences. In particular, we will discuss the similarities and differences of work on negotiation models in economics and operations research vis a vis work in computer science. Additionally, we will present future beneficial synergies between the two research communities so that more effective prescriptive models as well as ways to provide advice and decision support to decision makers can be constructed.

The rest of the chapter is organized as follows. Section 2 introduces different perspectives of formal negotiation research, highlighting the strengths and weaknesses of different approaches. We then propose a framework for negotiation reasoning in Section 3, which consists of five types of reasoning, namely, reasoning about negotiation procedure, reasoning about problem structure, reasoning about claiming/creating value, reasoning about persuasion, and tactical reasoning. Sections 4 through 8 elaborate these five different levels of reasoning, not only providing both overviews of existing research, but also pointing out ways to making negotiation modeling and analysis closer to real life. Section 9 concludes this chapter by reviewing different issues of reasoning in negotiation, and proposing new directions of negotiation research.

\section{Formal Negotiation Research: Different Perspectives}

We have stated in the introduction that quantitative negotiation research can be divided into two sides: analytic and computational research. While the former group focuses on rigorous mathematical analysis, the latter seeks to create computationally tractable formal models, as well as, design and implementation of negotiation systems under various application scenarios. The connection between analytic and computational research is important: analytic models can provide valuable managerial insights, and help choose a suitable bargaining protocol in the face of difficult

tasks. Computational research is invaluable in developing (a) heuristic approximate solutions to analytic models of high computational complexity, (b) aiming to incorporate additional factors of realistic negotiations, such as argumentation, negotiation context or culture and (c) provide decision support systems and bargaining protocols in situations where analytic techniques cannot offer practical guidance. 
Both negotiation process and negotiation outcome must be addressed for realistic modeling. However, most of the existing research has focused on how to achieve outcomes with particular desirable properties, for example Pareto optimality, or equilibrium behavior. Analytic negotiation research has focused on negotiation outcome rather than process due to the game-theoretical approaches they adopt: by assuming full rationality and various simplifying settings, bargaining game models can lead to highly stylized equilibrium analysis, which can be used to predict the outcome. Such approaches have been under increasing attack (e.g., (Neelin, Sonnenschein, and Spiegel 1988), (Ochs and Roth 1989), (Sebenius 1992)) for the rigidity and unrealistic assumptions of the game theoretic models. Even as early as the 1980s there were spirited debates between game theorists (e.g. Harsanyi) and other scientists (e.g. Kadane, Larkey, Roth and others) that adopted non-equilibrium game theory, bounded rationality, proclaimed the existence of subjective prior distributions on the behavior of other players and urged the use of Bayesian decision-theoretic orientations (interested readers are referred to (Kadane and Larkey 1982a), (Kadane and Larkey 1982b), (Harsanyi 1982a), (Harsanyi 1982b), (Kahan 1983), (Roth and Schoumaker 1983), (Rothkopf 1983) and (Shubik 1983)).

In contrast to research focusing on generation of outcomes, other research has focused primarily on the negotiation process (e.g., (Balakrishnan and Eliashberg 1995), (Zeng and Sycara 1998) and (Bac 2001)). The negotiation process refers to the events and interactions that occur between parties before the outcome and includes all verbal and non-verbal exchanges among parties, the enactment of bargaining strategies and the external and situational events that influence the negotiation. Process analysis in bargaining has mainly focused on either the back and forth exchanges between the negotiators or on the broader phases of strategic activity over time. The most general categorization that comes from such analysis of negotiation outcomes and processes is the distinction between competitive and cooperative situations $^{2}$, which is also referred to as distributive vs. integrative or hard vs. soft bargaining. In competitive negotiation each party seeks to maximize his own gain or maximize the difference in gains between himself and the other parties. On the other hand, in cooperative negotiation each party aims to increase joint gains (i.e. each party is both self focused and also other focused). Another view of distributive and integrative negotiations is that distributive negotiation can be regarded as a zero sum game where a fixed resource is simply divided whereas in integrative negotiation, interests of both parties are satisfied although there may be concessions on both sides.

Most recently, researchers (e.g., (Weingart, Bennett, and Brett 1993) and (Adair and Brett 2005)) have postulated that negotiations and negotiators do not fit neatly into cooperative or utility maximizing types but they are usually mixed-motive. In a mixed-motive interaction parties use a mixture of competitive and cooperative strategies to pursue their interests which usually are competing and compatible at the same time. Additionally, it has been observed in the literature (e.g., (Thompson 1996)) that negotiating on a single issue typically leads to distributive negotiation

2 The words cooperative and competitive here are not to be confused with the notions of cooperative and non-cooperative game theory. 
whereas in multi-issue negotiations, tradeoffs among the different issue values and the differential importance of issues to the parties enable integrative processes and outcomes. Negotiation with multiple issues is so complex that defies rigorous modeling using non-cooperative game theory. Therefore, some researchers have studied multi-issue negotiations using issue by issue negotiation and analyze when this simplification is applicable (e.g., (Luo et al. 2003)). In cooperative game theory, Nash and others ((Nash 1951), (Nash 1953), (Luce and Raiffa 1989), (Kalai and Smorodinsky 1975) and (Ponsati and Watson 1997)) have focused on designing appropriate axioms that characterize the negotiation solution.

Although game-theory has been the underlying fundamental theory behind many of the analytic models, its explanatory and prescriptive merits have long been debated for the following reasons: First, standard assumptions in various gametheoretic models are incompatible with real-life situations. Among the restrictive reasons are 1) the rules of the games and beliefs of the players are common knowledge", 2) players have infinite reasoning and computational capacity to maximize their expected payoffs given their beliefs of others' types, behaviors and beliefs. Second, equilibrium analysis tends to focus on negotiation outcome yet overlooks the negotiation process. Third, information disclosure mechanisms, i.e., who knows what under which conditions, which affect the negotiation process and outcomes in real life situations, are difficult to model. Allowing partial information, instead of either complete information or no information, poses a daunting challenge for multi-period game-theoretic analysis. This is still true even if agents have perfect reasoning powers. Fourth, most game-theoretic models assume that agents are fully rational, while in practice people are not, and they hence do not employ equilibrium strategies. Even if players are assumed to be perfectly rational, Roth and his co-workers ((Roth and Malouf 1979), (Roth et al 1981) and (Roth and Murnighan 1982)) have shown in experiments with human subjects that subjective expectations of the players might influence the outcome, contrary to game theoretic assumptions. Interested readers are referred to (Lai et al. 2004) for a comprehensive review of game-theoretic negotiation models.

\section{A Framework for Negotiation Reasoning}

In the following we will concentrate on multi-issue negotiation since this is the most realistic and challenging. The elements of negotiations have been identified as negotiation parties, negotiation context, negotiation process, and negotiation outcomes in (Agndal 2007). Such elements are viewed in a static way in most of the business negotiation research. We believe, however, that the purpose of reasoning in negotiation is essentially managing such elements dynamically over time such that the negotiation process moves towards each partys desired outcome. We proposal five types of reasoning based on the object being managed. The relationships between the different types of reasoning and negotiation elements are shown in Figure 1. 


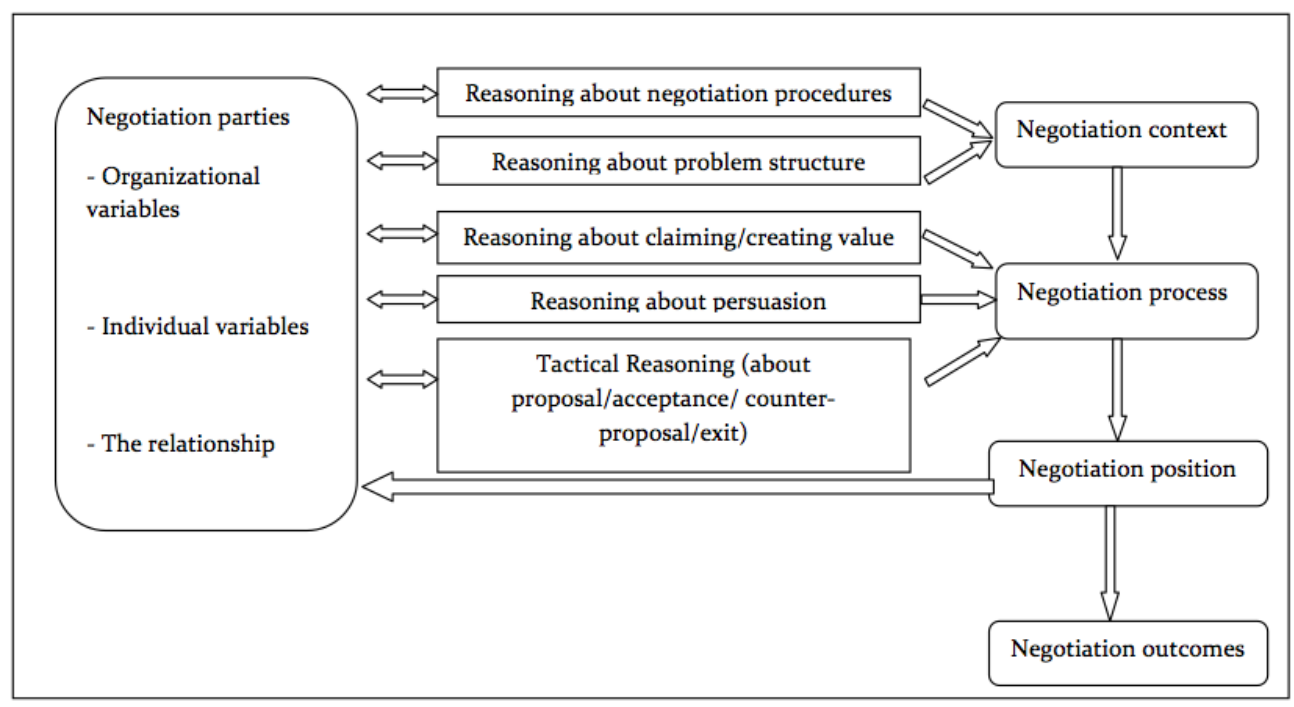

Fig. 1 A framework for reasoning in negotiation

(a) Reasoning about negotiation procedures. While the negotiation procedures, i.e., what and how to negotiate, are usually given, it is sometimes determined by the negotiation parties either before or during the negotiation. This is especially true in the presence of multiple issues, incomplete information, and a changing environment. On the one hand, the negotiation procedures can be viewed as a strategic control variable from each negotiators point of view. On the other hand, each negotiators preference over different procedures indirectly conveys information about his social motives.

(b) Reasoning about problem structure. The problem structure in a negotiation problem is defined as negotiation goals and issues, relations and constraints among the variables and reservation prices that denote the minimum acceptable levels at which constraints can be satisfied. [Sycara, 1991] To avoid deadlocks in a negotiation and make sure that agreements are reached, problem restructuring is an effective tool in managing the negotiation context as well as negotiation parties goals, beliefs and relationships. In some sense, concession-making during negotiation can be viewed as an embryonic form of problem restructuring.

(c) Reasoning about claiming/creating value. While value creating is about how to make the pie bigger, value claiming is about how to get a larger proportion of the pie. How negotiators reason between claiming and creating value has much to do with their social motives, as well as the negotiation context, e.g., the deadline effect, and BATNA (Best alternative to a negotiated agreement).

(d) Reasoning about persuasion. Negotiation is not just about proposal and counterproposal. In real-life negotiations, it is of crucial importance to be able to persuade others, i.e., to influence how other people reason about different alternatives. 
(e) Tactical Reasoning about proposal/acceptance/counter-proposal/exit. Proposal, acceptance, counter-proposal, and exit constitute basic elements of the negotiation protocol.

It is worth pointing out that all of the above categories of reasoning stem from the negotiation parties internal variables, and they affect the negotiating parties as a consequence. Consider, for instance, that each party might have prior knowledge of its opponents belief structure, such understanding can be updated as the consequence of either his own learning through dealing with his opponent, or his opponents adopting of persuasion.

\section{Procedures for Multi-Issue Negotiation}

Faced with multiple issues, agents need to decide two concerns before the negotiation: one is the kind of negotiation procedure (agenda) they will take and the other is the type of agreement implementation. There usually exist three types of negotiation procedures: separate, simultaneous and sequential ((Inderst 2000), (Gerding et al, 2000)). Separate negotiation means agents negotiate each issue separately (independently and simultaneously as if there are $\mathrm{n}$ pairs of representatives for the two agents, and each pair independently negotiates one issue). Simultaneous negotiation means two agents negotiate a complete package on all issues simultaneously. Sequential negotiation is when two agents negotiate issue by issue sequentially, i.e. issue-by-issue negotiation. In issue-by-issue negotiation, agents also need to decide the order to negotiate each issue.

There can be two types of agreement implementation: sequential and simultaneous. Sequential implementation means the agreement on each issue is implemented once it is reached, while simultaneous implementation is that agreements are implemented together when all issues are settled.

Research on issue-by-issue negotiation is mostly based on Rubinsteins bargaining model (dividing a single pie) by introducing another issue (pie). The two issues may have different values and be differentially preferred by the agents. Besides, the two issues can either be simultaneously available or arrived at in a sequential order.

Negotiating issues simultaneously is very challenging both for people and for automated models. The difficulties are due to bounded rationality: Simultaneously negotiating a complete package might be too complex for individual agents. However, this reason only provides an intuitive idea on issue-by-issue negotiation. More theoretical explanation or implication is needed. Next, we review theoretical work on why issue-by-issue negotiation may arise in two different contexts: incomplete information and complete information.

Signaling might be the first and only reason that researchers mention, why issueby-issue negotiation arises under incomplete information. (Bac and Raff 1996) study a case with two simultaneous and identical pies where agents can either choose sequential negotiation with sequential implementation or simultaneous negotiation with simultaneous implementation. The authors show that in the context of com- 
plete information agents will take simultaneous negotiation and reach an agreement without delay. But in the context of asymmetric information (assume two players A and $\mathrm{B}, \mathrm{A}$ is informed, but $\mathrm{B}$ is uncertain of As time discount, which can take one of the values: $\delta_{H}$ with probability $\pi$ and $\delta_{L}$ with $1-\pi$ ), the authors argue that when Bs time discount is in some interval (not so strong and also not so weak), the strong type of the informed agent (A with $\delta_{H}$ ) may make a single offer on one pie and leave it to the opponent (B) to make an offer on the second pie, while a weak type of informed player (A with $\delta_{L}$ ) only makes a combined offer. So if issue-by-issue negotiation arises, it is because the strong and informed agent, by a single (signaling) offer, wants to let her opponent know she is strong and make the opponent concede.

(Busch and Horstmann 1999) similarly but more strictly study the signaling factor with an incomplete information model that allows for different sized pies and each kind of agreement implementation. By setting some parameter configurations, they show that issue-by-issue negotiation may arise with signaling and they prove under such configurations signaling does not arise if agents can only bargain a complete package. So the authors argue issue by issue negotiation arises purely because some favorable endogenous agenda for issue-by-issue bargaining is available. Besides, they also show that if issue-by-issue bargaining arises agents will negotiate the large pie first.

As mentioned above, under complete information agents will negotiate a complete package if it is with simultaneous and identical pies. But when assumptions are changed, issue-by-issue negotiation could possibly arise under complete information.

(Busch and Horstmann 1999) study the difference between incomplete contract (issue-by-issue) and complete contract (simultaneous) negotiation with sequential pies on which agents have different preferences. From the equilibrium outcomes of the two procedures, it is shown that if agents are heterogeneous, they might have conflicting preferences on the two procedures, which means one prefers incomplete contract procedure but the other may prefer complete contract procedure. Further, Busch and Horstmann also show that when time is costless agents will agree to negotiate a complete contract, while if time is very valuable agents will negotiate an incomplete contract. From a different perspective, (Lang and Robert W. Rosenthal 2001) argue that joint concavity of two agents payoffs can eliminate the possibility of non-fully-bundled (issue-by-issue) equilibrium offers, but in realistic settings, the property of joint concavity usually is not true so that a partial bundled offer on a subset of unsettled issues may be superior over a fully-bundled offer.

Additionally, the occurrence of breakdown can impact a multi-attribute negotiation. Sometimes agents insisting on some issue may lead the whole negotiation to breakdown. (Chen 2006) studied issue-by-issue negotiation taking into consideration the probability of breakdown. Chen applies a probability setting that a negotiation breaks down if a proposal on some issue is rejected. He assumes that agents utility functions are linear additive so that breakdown on one issue does not affect others. By comparing the equilibrium outcomes between issue-by-issue negotiation and simultaneous negotiation, Chen argues that when the probability of breakdown is low, agents prefer to negotiate a complete package because intuitively they know 
that the bargaining can last long enough so that agents can get to a Win-Win solution with inter-issue tradeoffs. However, when the breakdown probability is high, agents weakly prefer issue-by-issue negotiation. Chen also shows that if agents are sufficiently heterogeneous, issue-by-issue negotiation may also be superior over simultaneous negotiation. (In and Serrano 2004) assume that the negotiation breakdown of one issue can make the whole negotiation fail, and agents are restricted to making an offer on only one of the remaining issues each round. They show that when the probability of breakdown goes to zero, there is a large multiplicity of equilibrium agreements and therefore inefficiency arises. But it does not happen for simultaneous negotiation. However, if agents are not restricted to making offers on only one issue at each round (i.e. agents can make partially or fully bundled offers), the outcome turns out to be Pareto-efficient (In and Serrano 2003). Thus, In and Serranos work indicates strict issue-by-issue negotiation may increase inefficiency. (Inderst 2000) might be the only work that compares those three different negotiation procedures in one paper. On a set of unrelated issues, Inderst argues that if the issues are mutually beneficial, agents will prefer to bargain simultaneously over all issues.

Besides the work above, (Weingart, Bennett, and Brett 1993) studies the multiattribute negotiation problem within a specific context allowing "Selective Acceptance". In such a context, the offer initially needs to be a complete package including all issues, but agents can accept or reject the whole package as well as selectively accept part of the package on some issues. But if agents accept a part on some issues, these issues can not be reopened again. The author indicates that in some situations this leads to good solutions. Weinberger shows Selective Acceptance can lead to inefficient equilibrium outcomes if some issues are indivisible or agents have opposing valuations on issues. For comparison, Weinberger shows that inefficient outcomes do not arise under the rule only to accept or reject the whole package. However, the equilibrium outcomes with Selective Acceptance are not dominated by the efficient outcome. It means there must be some agent who is better off by the rule of Selective Acceptance and will not agree on the efficient outcome.

In the computational literature, (Fatima, Wooldridge, and Nicholas R. Jennings 2004a) and (Fatima, Wooldridge, and Jennings 2004b)propose an agenda-based framework for multi-attribute negotiation. In their framework, the agents can propose either a combined offer on multiple issues or a single offer on one issue. Different from the game theoretic models, their work focuses on computational tractability. They assume that the agents adopt time-dependent strategy and the agents may make decisions on the issues independently faced with a combined offer. For example, if there are two issues in a combined offer, say $x_{1}$ and $x_{2}$, an agent may have two independent strategies $S_{1}$ and $S_{2}$ which are used to decide whether to accept $x_{1}$ and $x_{2}$. They make the assumption that the agents utility functions are given before the negotiation and they are linear additive. Pareto-optimality is not addressed. 


\section{Changing the Structure of the Negotiation Problem}

Problem structure refers to "characteristics of their feasible settlement spaces and efficient frontiers" (Mumpower 1991). As pointed out in (Mumpower 1991), while some problem structures lead to agreements with efficient outcomes, others lead to inefficient outcomes, or deadlocks. Negotiation restructuring, therefore, seeks to understand the situation and perception of the negotiators, and finds favorable directions to change the agents perceptions of the interaction, and hence the decisive factors of the negotiation.

Negotiation restructuring is an effective tool for all sides in a negotiation so as to achieve joint gains by enlarging the pie. Very often, a third party mediator may be engaged to facilitate the negotiation and break deadlocks. A mediator can manage the negotiation environment so as to break or avoid deadlocks. (Sycara 1991) proposed the concept of problem restructuring, i.e., to dynamically change the structure of the negotiation problem to achieve movement towards agreement. Under the context of her PERSUADER automated negotiation system, Sycara put forward four types of problem structuring: (1) introduction of new goals, (2) goal substitution, (3) goal abandonment, and (4) changing the reservation prices of the negotiating parties. Sycara also provides four methods to achieving the directions of problem restructuring, namely, 1) Case-based reasoning (utilizing previous cases and experiences of dispute resolution), 2) Situation Assessment (representing and recognizing negotiation problems in terms of their abstract causal structure), 3) Graphic search and control (Search for correlations amongst an agents goals in agents' goal graphs), 4) Persuasive argumentation (generating various arguments, e.g., threats and promises). (Shakun 1991) developed another framework of negotiation problem restructuring, namely ESD (evolutionary systems design), which involves "evolution of the problem representation to an evolved structure that is not equivalent to the original one." The authors implemented the ideas in various scenarios including labor relations and buyout in the airline industry. (Kersten et al. 1991) introduce a rule-based restructurable negotiation model characterizing the hierarchy of each negotiating agents goals, and propose ways to restructuring the negotiation problem.

\section{Value Claiming and Value Creating}

Agents negotiate with certain motivations in mind. Many social science papers have adopted the somewhat rough distinction between selfish and prosocial motivation (see (Weingart, Bennett, and Brett 1993) for example). Selfish motivation is characterized by competitive and individualistic goals, while prosocial motivation is characterized by cooperative and altruistic goals. Admittedly, in a realistic setting, a negotiator has mixed motives rather than behave purely selfishly or purely prosocially. This framework, however crude it may be, has been well accepted in the social sciences community. 
The agents social motives give rise to different behaviors during negotiation. Referred to as "win-win", "variable sum" or "integrative" in various works, valuecreating is the process wherein the negotiating parties work together to resolve conflicts and achieve maximum joint benefits. By contrast, value-claiming behavior is often referred to as "win-lose", "fixed sum" or "distributive". A value-claiming negotiating agent targets individual utility maximization without joint gains improvement.

(Paruchuri et al. 2009) is a first attempt to analytically model agents social motives and their potential adaptation during the negotiation process. They model agents selfish/prosocial motivation as part of the state in a Partially-Observable Markov Decision Process (POMDP) model, and provide possibilities for negotiating agents to learn each others social motives through interactive moves. Each negotiator has a mental model consisting of both himself and the other players, allowing him to update his beliefs about his opponents social motives through observations as well as allowing him to generate corresponding moves so as to influence other negotiators belief structures.

In more traditional modeling settings, an agents motivation is usually to maximize its own utility. The utility function or preferences structure of negotiating parties for different interests and issues, i.e., how they trade-off or prioritize different issues, provides the ultimate driving force for the decision making in a negotiation process. Given a set of issues, interests, and positions, a utility function or preference relation specifies how agents evaluate different alternatives. Most of the literature about negotiation provides a static and crisp definition of the utility function. In contrast, (Fogelman-Soulie, Munier, and Shakun 1983) develop an MDP model for the problem of bilateral two-issue negotiation. Instead of assuming bivariate utilities, the one-stage payoff is expressed as a payoff probability distribution representing the probability that a player obtains various amounts of each of the two variables. (Kraus, Wilkenfeld, and Zlotkin 1995) discuss different forms of continuous utility functions over all possible outcomes, e.g., time constant discount rates and constant cost of delay. (Zlotkin and Rosenschein 1996) present an approach to the negotiation problem in non-cooperative domains wherein agents' preferences over different intermediate states are captured by "worth functions" by considering the probabilistic distance between intermediate states and final states. (Rangaswamy and Shell 1997) design a computer-aided negotiation support system, one part of which is to help negotiating parties disaggregate their own preferences and priorities in order to understand them better, utilizing several utility assessment techniques.

(Faratin, Sierra, and Jennings 2002) use a given linearly additive multi-attribute utility function to represent agent preferences. Each agent is assumed to have a scoring function that gives the score it assigns to a value of each decision variable in the range of its acceptable values. Then the agent assigns a weight to each decision variable to represent its relative importance.

A number of papers represent the trade-off between multiple issues using constraints instead of utility functions. As a representative example, (Balakrishnan and Eliashberg 1995) propose a single-issue negotiation process model where the utilities are simply the negotiation outcome, and agents' dynamic preferences are 
represented using a constraint with the left-hand side denoting agents' "resistance forces", and right-hand side "concession forces".

We identify in general three inherent driving forces behind negotiators tradingoff decisions between value-claiming and value-creation. First, different negotiators have different social motives. While some agents are selfish, others are prosocial. The inherent agent characteristics largely determine the nature of the negotiation. Second, there exists so-called "deadline effect", i.e., as the deadline of the negotiation approaches, agents make more efforts to create higher incremental value. This could be explained by the fact that agents could create more value at later negotiation rounds based on what has been achieved in the previous rounds (Zartman and Berman 1983). (Bac 2001) builds a different analytic model and argues that deadline effect happens because the costs and benefits of negotiation efforts are not synchronized: while efforts are incurred in the negotiation rounds, the benefits are only realized after the final round. Third, the evolving BATNA (best alternative to negotiation agreement) is also behind agents trading-off behaviors. This is especially relevant in the presence of dynamic uncertain availability and quality of outside options. ( $\mathrm{Li}$, Giampapa, and Sycara 2006) build a bilateral negotiation model with the stochastic, dynamic outside options. The negotiation strategies are affected by outcome through their impact on the reservation price. Three modules with increased complexity, namely single-threaded negotiations, synchronized multi-threaded negotiations, and dynamic multi-threaded negotiations, are studied. In the single-threaded negotiation model, optimal negotiation strategies are determined without specifically considering outside options. Then the synchronized multi-threaded negotiation model addresses concurrently existing outside options. The dynamic multi-threaded negotiation model further extends the synchronized multithreaded model by considering the dynamic arriving future outside options. Experimental studies show that the agent can achieve significant utility increase if she takes outside options into consideration, and the average utility is higher when her negotiation decision-making addresses not just the concurrent outside options, but foresees future options.

\section{Fair Division ${ }^{3}$}

There is also some research on multi-attribute negotiations that focuses on the concept of "fair-division" and develops division procedure from the perspective of cooperative game theory. Usually, the goal of the procedure is to fairly divide a set of items between two agents, and it can consist of two steps: the first step ensures an efficient outcome and the second step establishes "fairness" through a redistribution of gains.

This approach was first developed by Knaster and Steinhaus based on the idea of auctions (Raith 2000). The Knaster procedure is quite simple. In the first step, all items are assigned to the "winner" who totally values the items most, and then "fairness" is established through monetary transfers. The idea is two agents fairly share the excess. Knasters procedure focuses on fairly sharing of the excess between agents, but the percent of estate of the two agents is not fair. With such a

${ }^{3}$ Interested readers are referred to Chapter 11 by Klamler in this volume for a comprehensive survey of various approaches to fair division. 
consideration, (Brams and Taylor 1996) introduce another fair-division procedure named "Adjusted Winner", which implements an equitable outcome. In this procedure, each item (not all items as in Knasters procedure) is assigned to the agent who values it most in the first step, and then some money is transferred from the temporary winner to the temporary loser in the second step such that the percent of estate between agents is the same. (Raith 2000) points out the outcome of "Adjusted Winner" might not be efficient. Thus Raith designs another approach named "Adjusted Knaster" based on both of them, which marries Knasters efficient adjustment with the equitability condition of "Adjusted Winner". Raith also compares the outcomes of "issue-by-issue" negotiation and "package deals", and indicates the former might not be efficient.

\section{Persuasion for Conflict Resolution}

In a general sense, negotiation can be viewed as "planning other agents plans" (Sycara 1989), i.e., to use persuasive argumentation to influence the other sides belief structure. The purpose of using such argumentation is to influence the other partys utility function, which derives from his belief structure, including goals, importance attached to different goals, and relations between goals. The associated reasoning involves not the priorities of different issues and interests, but also the graphic structure, i.e., how one goal affects another. To put it simply, we can either change the opponents utility value of one objective, or change the relative importance he assigns to that objective.

(Sycara 1990b) is the first published work to incorporate argumentation into negotiation, and to illustrate the merit of argumentation-based reasoning in negotiation dialogues. Sycara also proposes a concrete framework in the light of a negotiation support system. (Kraus, Sycara, and Evenchik 1998) formalize the above argumentation tools and protocols in a set of logic models. They present a mental model representing agents beliefs, desires, intentions, and goals. Argumentation is modeled as an iterative process in the sense that it is initiated from agent exchanges and then changes the negotiation process, hopefully toward cooperation and agreements. Their logic models help specify argument formulation and evaluation. Other argumentation-based negotiation frameworks include (Parsons and Jennings 1996) and (Tohm 2002). (Amgoud, Dimopoulos, and Moraitis 2007) points out that the inherent weakness of the above-mentioned frameworks lies in that they cannot explain when argumentations can be used in negotiation, and how they are dealt with by the agents who receive them. They establish a unified framework which formally analyzes the role of argumentation, and especially addresses how agents respond to arguments.

Argumentation can also be combined with additional factors relevant to the negotiation process. (Karunatillake et al. 2009) present a framework allowing agents to argue, negotiate, and resolve conflicts relating to their social influences within a multiagent society. Their framework can be used to devise a series of concrete al- 
gorithms that give agents a set of argumentation-generation strategies to argue and resolve conflicts in a multi-agent task allocation scenario, especially when the social structure is complicated to analyze in other ways. They show that allowing agents to negotiate their social influences presents an effective and efficient method that enhances their performance within a society.

\section{Tactic Reasoning}

In this section, we provide an overview of modeling efforts in externally-observable behavior and characteristics such as strategies, tactics and outcomes of negotiation. In the computational field, the existing work mainly focuses on automated negotiation frameworks and tractable heuristics.

Sycara ((Sycara 1990b), (Sycara 1990a), (Sycara 1991)) uses a case-based reasoning approach for multi-attribute negotiations where the agents make offers based on similarity of the negotiation context (including issues, opponents, and environment) to previous negotiations. Sycara also uses automatically generated persuasive argumentation as a mechanism for altering the utilities of agents, thus making them more prone to accept a proposal that otherwise they might reject.

Most of the existing research focuses on agents' optimal actions based on their reasoning strategies, and the efficiency compared to Pareto optimal solutions or human negotiation outcomes. (Faratin, Sierra, and Jennings 2002) provide conditions for convergence of optimal strategies, and negotiation outcomes for different scenarios with linear utility functions. (Lai, Sycara, and Li 2008) propose a protocol where agents negotiate in a totally decentralized manner, have general non-linear utility functions in multi-issue negotiation aiming at reaching Pareto optimal outcomes. The agents have non-linear and inter-dependent preferences and have no information about the opponents preference or strategy. The authors show that their model is computationally tractable and the outcomes are very close to Pareto equilibrium results.

An important issue in multi-attribute negotiation is the tradeoff process between self-interested agents on different issues. (Faratin, Sierra, and Jennings 2002) propose a novel idea to make the agents trade off on multiple issues. They suggest that the agents should apply similarity criteria to trade off the issues, i.e., make an offer on their indifference curve which is most similar to the offer made by the opponent in the last period. However, in this approach, to define and apply the similarity criteria, it is essential that the agents have some knowledge about the weights the opponent puts on the issues in the negotiation. A subsequent work (Coehoorn and Jennings 2004) proposes a method based on kernel density estimation to learn the weights. But the performance still might be compromised if the agents have no or very little prior information about the real weights the opponent assigns on the issues. Moreover, it will be difficult to define and apply the similarity criteria if the agents utility functions are nonlinear and the issues are interdependent. 
(Luo et al. 2003) develop a fuzzy constraint based framework for multi-attribute negotiations. In this framework, an agent, say the buyer, first defines a set of fuzzy constraints and submits one of them by priority from the highest to lowest to the opponent, say a seller, during each round. The seller either makes an offer based on the constraints or lets the buyer relax the constraints if a satisfactory offer is not available. The buyer then makes the decision to accept or reject an offer, or to relax some constraints by priority from the lowest to highest, or to declare the failure of the negotiation.

( $\mathrm{Li}$ and Tesauro 2003) introduce a searching method based on Bayesian rules. It is assumed that the agents have some prior knowledge about the opponents utility function. When they concede, the agents apply depth-limited combinatorial searching based on their knowledge to find a most favorable offer. If the proposal is rejected then the agents update their knowledge by Bayesian rules. Their work assumes that the agents know partially about the opponents utility function and the work does not address Pareto-optimality.

There also exists some research that addresses multi-attribute negotiations on binary issues. For instance, (Robu, Somefun, and Poutr 2005) propose an approach based on graph theory and probabilistic influence networks for the negotiations with multiple binary issues; (Chevaleyre, Endriss, and Maudet 2005) address a categorization problem of the agents utility functions under which the social optimal allocation of a set of indivisible resources (binary issues) is achievable.

(Zeng and Sycara 1998) develop an automated negotiation model wherein agents are capable of reasoning based on experience and improve their negotiation strategies incrementally. They utilize the Bayesian framework to update an agent's belief about its opponents. (Lin et al. 2008) model an agent's internal reasoning in terms of generating and accepting offers. When generating offers, an agent selects the best offer among the offers that the agent believes might be accepted. To be more specific, the agent selects the minimum value of 1) the agent's own estimation of the offer and 2) the agent's estimation of its opponents' acceptable offer, under the pessimistic assumption that the probability that an offer is accepted is based on the agent that favors the offer the least. In discussing the agent's reasoning about accepting offers, they make the assumption that each offer is evaluated based on their relative values compared to the reservation price.

We summarize the existing research as follows. First, almost all the models in the existing research are based on the assumption that the agents in a negotiation have explicit utility functions. Some also assume that the agents completely or partially know their opponents utility function. Second, the existing models either assume a simple utility function (two issues with linear additive utility functions) or focus on binary issue or cooperative negotiations. Finally, Pareto-optimality and tractability have not been considered simultaneously in most of the models.

\section{Third Party Mediation}

There are some papers that adopt a non-biased mediator in the negotiation. (Ehtamo et al. 1999) present a constraint proposal method to generate Paretofrontier of a multi-attribute negotiation. The mediator generates a constraint in each step and asks the agents to find their optimal solution under this constraint. If the 
feedback from the agents coincide, then a Pareto optimal solution of the negotiation is found; otherwise, the mediator updates the constraint based on the feedback and the procedure continues. They show that their approach can generate the whole Pareto-frontier efficiently. In their work, the negotiation agents do not have the ability to make self interested decisions or have autonomous strategies, which limits its application in the negotiations with self-interested agents. Moreover, the approach relies on the assumption that the agents can solve multi-criteria-decision-making (MCDM) problems efficiently, which is not always the case in practice.

(Klein et al. 2001) proposes a mediating approach for negotiating complex contracts with more decision flexibility for the agents. Their approach focuses on the negotiations with binary valued issues ( 0 or 1$)$. The non-biased mediator generates an offer in each period and proposes to both agents. Then the agents vote whether to accept the offer based on their own strategies. If both agents vote to accept, the mediator mutates the offer (to change the values of some issues in the offer from 0 to 1 , or reverse) and repeats the procedure. If at least one agent votes to reject the offer, the mediator mutates the last mutually acceptable offer and repeats the procedure. This approach is difficult to be applied to problems with continuously-valued issues. Besides, a key assumption they make is that the mediator always can change the contract even if both agents have already voted to accept it, which might not be tractable in practice.

(Lai, Li, and Sycara 2006) presents a model with incomplete information, decentralized self-interested agents that is Pareto optimal. Each agent not only does not know the utility function of the opponent but also does not know her own. The authors assume that given a limited number of offers, an agent, though not having an explicit model of her preference, can compare them, and she can decide whether an offer is acceptable or not. A non-biased mediator is adopted in the model to help the players achieve Pareto optimality and overcome the difficulty of absence of information about the preferences of the agents. The approach reduces the negotiation complexity by decomposing the original n-dimensional negotiation space into a sequence of negotiation base lines. Agents can negotiate upon a base line with simple strategies. The approach is shown to reach Pareto optimal solutions asymptotically within logarithmically bounded computational time.

\section{Agents for Decision Support}

(Braun et al. 2006) summarize modeling approaches in decision-support negotiation literature, including 1) probabilistic decision theory, 2) possibilistic decision theory, 3) constraint-based reasoning, 4) heuristic search, 5) Bayesian learning, 6) possibilistic case-based reasoning, 7) Q-learning, 8) evolutionary computing. This classification is based on specific computational methods used in computerized system design, and can serve those readers who are interested in a complete review of operational analysis techniques in computational literature. However, a detailed review of decision support systems is outside the scope of the present chapter (but see Chapter 21 by Kersten and Chapter 23 by Schoop in this volume).

There has been consistent evidence that using an intelligent agent to negotiate with a human counterpart achieves better outcomes than negotiation between two human beings (see (Lin et al. 2008) and (Kraus et al. 2008) for example). While 
the results are encouraging, several complexities restrict their significance: 1) Implementation of the computational model. It remains challenging to elicit human preferences on multiple issues. 2) Information exchange mechanism. Computational negotiation agents might not be able to exchange information as efficiently as human beings in situations where accurate representations are hard to achieve. 3) How "efficient" the negotiation outcome is ultimately depends on human affect and cultural factors, which have not been taken into account in the existing computational literature.

\section{Conclusions}

In this chapter we gave a selective review of the analytic and computational research organized within a framework of the different types of reasoning that occur in negotiation. We compared and contrasted the achievements of both of these strands of research. The analytic research in general creates elegant and simplified models that provide insights and often formal guarantees about optimality or model behavior. The computation research focuses on how to make analytic models computationally tractable, increase their flexibility, and make the algorithms decentralized. In addition, the computational literature aims to incorporate additional factors in the analytic models thus making them more realistic. A parallel and very important aim of the computational negotiation research is to incorporate negotiation models into decision support systems or into systems that negotiate with humans. The aims of the two literatures are synergistic, espousing the long term goal of achieving analytic models with computational guarantees that incorporate elements of realistic negotiations.

The economic models of bargaining that dominated the field in its nascent stages posit that the ultimate aim in negotiation is maximizing one's own gain and the easiest and most efficient way to realize this aim is through integrative potential (Nash, 1953). However, it is now well-documented that pure economic outcomes are poor indicators of not only what people value in negotiation but also of their behavioral manifestations. Research has shown that perceptions of self, relationship with the other party or the desire to maintain a positive image may be as influential as, if not more, than economic gains. Issues such as self-efficacy, self-esteem, maintaining face or maintaining social relationships with the other party may be of critical concern to the negotiators and subsequently influence processes and outcomes ((Bandura 1977), (Snyder and Higgins 1988), (Anderson and Shirako 2008), (McGinn and Keros 2002)). The question of what negotiators value and how it influences their perceptions of the outcome has become a fertile area of bargaining research to the extent that (Curhan, Elfenbein, and Xu 2005) developed and validated a framework (the subjective value inventory (SVI)) subjective values to measure subjective value in negotiation. The authors also find that the SVI is a more accurate predictor of future negotiation decisions than economic outcomes, which demonstrates again that what people value in negotiation cannot be fully or accurately predicted by sole 
profit maximization models. Therefore a fertile area for future research would be to incorporate these subjective values into formal models. This will allow increased understanding for example of the conditions under which different subjective factors influence negotiations the most, different nonlinearities or tradeoffs among these subjective factors etc. Another related issue is validation of analytic and computational models. If the formal models were able to incorporate representations and reasoning schemes of cognitive factors, then human experimental data could be used to validate the models.

Another important future research direction is to study repeated interactions. Almost all of current research considers negotiation as a one-time event. However, in real life, negotiations are a repeated phenomenon, and very often they occur with the same individuals (e.g. in business negotiations). Currently, there is very limited research in repeated games and experience-based negotiation. We believe that analytic and computational models that incorporate repeated interactions and utilize machine learning techniques would be an important step in making negotiation less art and more science.

Acknowledgements The current research has been supported by the ARO Multi University Research Initiative grant W911-NF-0810301.

\section{References}

1. Adair, Wendi L., and Jeanne M. Brett. 2005. The Negotiation Dance: Time, Culture, and Behavioral Sequences in Negotiation. Organization Science 16, no. 1: 33-51.

2. Agndal, Henrik. 2007. Current trends in business negotiation research: An overview of articles published 1996-2005. Stockholm School of Economics, February.

3. Amgoud, Leila, Yannis Dimopoulos, and Pavlos Moraitis. 2007. A unified and general framework for argumentation-based negotiation. In Proceedings of the 6th international joint conference on Autonomous agents and multiagent systems, 1-8. Honolulu, Hawaii.

4. Anderson, Cameron, and Aiwa Shirako. 2008. Are individuals' reputations related to their history of behavior? Journal of Personality and Social Psychology 94, no. 2: 320-333.

5. Bac, Mehmet. 2001. On Creating and Claiming Value in Negotiations. Group Decision and Negotiation 10, no. 3 (May 1): 237-251.

6. Bac, Mehmet, and Horst Raff. 1996. Issue-by-Issue Negotiations: The Role of Information and Time Preference. Games and Economic Behavior 13, no. 1 (March): 125-134.

7. Balakrishnan, P. V. (Sundar), and Jehoshua Eliashberg. 1995. An Analytical Process Model of Two-Party Negotiations. MANAGEMENT SCIENCE 41, no. 2 (February 1): 226-243.

8. Bandura, Albert. 1977. Self-efficacy: Toward a Unifying Theory of Behavioral Change. Psychological Review 84, no. 2: 191-215.

9. Bichler, Martin. 2000. A Roadmap to Auction-Based Negotiation Protocols for Electronic Commerce. In 33rd Hawaii International Conference on System Sciences, 6:6018. Vol. 6.

10. Brams, Steven J., and Alan D. Taylor. 1996. Fair division. Cambridge University Press.

11. Braun, Peter, Jakub Brzostowski, Gregory Kersten, Jin Baek Kim, Ryszard Kowalczyk, Stefan Strecker, and Rustam Vahidov. 2006. e-Negotiation Systems and Software Agents: Methods, Models, and Applications. In Intelligent Decision-making Support Systems, 271-300.

12. Busch, Lutz-Alexander, and Ignatius J. Horstmann. 1999. Endogenous Incomplete Contracts: A Bargaining Approach. The Canadian Journal of Economics / Revue canadienne d'Economique 32, no. 4 (August): 956-975. 
13. Carnevale, Peter J.D., and Edward J. Lawler. 1986. Time Pressure and the Development of Integrative Agreements in Bilateral Negotiations. The Journal of Conflict Resolution 30, no. 4 (December): 636-659.

14. Chen, M. Keith. 2006. Agendas in Multi-Issue Bargaining: When to Sweat the Small Stuff. Harvard Department of Economics.

15. Chevaleyre, Yann, U Endriss, and N Maudet. 2005. On maximal classes of utility functions for efficient one-to-one negotiation. In Proceedings of the 19th International Joint Negotiating Socially Optimal Allocations of Resources Conference on Artificial Intelligence (IJCAI2005: 941-946.

16. Coehoorn, Robert M., and Nicholas R. Jennings. 2004. Learning on opponent's preferences to make effective multi-issue negotiation trade-offs. In Proceedings of the 6th international conference on Electronic commerce, 59-68. Delft, The Netherlands: ACM.

17. Conlin, Michael, and Taiji Furusawa. 2000. Strategic Delegation and Delay in Negotiations over the Bargaining Agenda. Journal of Labor Economics 18, no. 1 (January 1): 55-73.

18. Curhan, Jared R., Hillary Anger Elfenbein, and Heng Xu. 2005. What do People Value when they Negotiate? Mapping the Domain of Subjective Value in Negotiation. Massachusetts Institute of Technology (MIT), Sloan School of Management, July.

19. Dreu, Carsten K. W. De. 2003. Time pressure and closing of the mind in negotiation. Organizational Behavior and Human Decision Processes 91, no. 2 (July): 280-295.

20. Ehtamo, Harri, Raimo P. HŁmŁlŁinen, Pirja Heiskanen, Jeffrey Teich, Markku Verkama, and Stanley Zionts. 1999. Generating Pareto Solutions in a Two-Party Setting: Constraint Proposal Methods. Management Science 45, no. 12 (December): 1697-1709.

21. Faratin, P., C. Sierra, and N. R Jennings. 2002. Using Similarity Criteria to Make Issue TradeOffs in Automated Negotiations. ARTIFICIAL INTELLIGENCE 142: 205-237.

22. Fatima, Shaheen, Michael Wooldridge, and Nicholas R. Jennings. 2004a. Optimal Negotiation of Multiple Issues in Incomplete Information Settings. In Third International Joint Conference on Autonomous Agents and Multiagent Systems, 3: Vol. 3.

23. Fatima, Shaheen S., Michael Wooldridge, and Nicholas R. Jennings. 2004b. An agenda-based framework for multi-issue negotiation. Artif. Intell. 152, no. 1: 1-45.

24. Fogelman-Soulie, Francoise, Bertrand Munier, and Melvin F. Shakun. 1983. Bivariate Negotiations as a Problem of Stochastic Terminal Control. MANAGEMENT SCIENCE 29, no. 7 (July 1): 840-855.

25. Froncek, Dalibor. 2009. Oberwolfach rectangular table negotiation problem. Discrete Mathematics 309, no. 2 (January): 501-504.

26. Gerding, Enrico H., David D.B. Bragt, and J. A. L Poutre. 2000. Scientific approaches and techniques for negotiation. A game theoretic and artificial intelligence perspective. CWI (Centre for Mathematics and Computer Science). ACM.

27. Harsanyi, John C. 1982a. Subjective Probability and the Theory of Games: Comments on Kadane and Larkey's Paper. Management Science 28, no. 2 (February): 120-124.

28. Harsanyi, John C.. 1982b. Rejoinder to Professors Kadane and Larkey. Management Science 28, no. 2 (February): 124-125.

29. In, Younghwan, and Roberto Serrano. 2003. Agenda restrictions in multi-issue bargaining (II): unrestricted agendas. Economics Letters 79, no. 3 (June): 325-331.

30. In, Younghwan, and Roberto Serrano. 2004. Agenda restrictions in multi-issue bargaining. Journal of Economic Behavior \& Organization 53, no. 3 (March): 385-399.

31. Inderst, Roman. 2000. Multi-issue Bargaining with Endogenous Agenda. Games and Economic Behavior 30, no. 1 (January): 64-82.

32. Kadane, Joseph B., and Patrick D. Larkey. 1982a. Subjective Probability and the Theory of Games. Management Science 28, no. 2 (February): 113-120.

33. Kadane, Joseph B., and Patrick D. Larkey.. 1982b. Reply to Professor Harsanyi. MANAGEMENT SCIENCE 28, no. 2 (February 1): 124.

34. Kahan, James P. 1983. On Choosing between Rev. Bayes and Prof. Von Neumann. Management Science 29, no. 11 (November): 1334-1336.

35. Kalai, Ehud, and Meir Smorodinsky. 1975. Other Solutions to Nash's Bargaining Problem. Econometrica 43, no. 3: 518, 513. 
36. Karunatillake, Nishan C., Nicholas R. Jennings, Iyad Rahwan, and Peter McBurney. 2009. Dialogue games that agents play within a society. Artificial Intelligence 173, no. 9-10 (June): 935-981.

37. Kersten, Gregory E., Wojtek Michalowski, Stan Szpakowicz, and Zbig Koperczak. 1991. Restructurable Representations of Negotiation. Management Science 37, no. 10 (October): 1269-1290.

38. Kim, Jeong-Yoo. 1996. Cheap Talk and Reputation in Repeated Pretrial Negotiation. Rand Journal of Economics 27, no. 4: 787-802.

39. Klein, Mark, Peyman Faratin, Hiroki Sayama, and Yaneer Bar-yam. 2001. Negotiating Complex Contracts.

40. Kraus, Sarit. 1997. Negotiation and cooperation in multi-agent environments. Artificial Intelligence 94, no. 1-2 (July): 79-97.

41. Kraus, Sarit, Penina Hoz-Weiss, Jonathan Wilkenfeld, David R. Andersen, and Amy Pate. 2008. Resolving crises through automated bilateral negotiations. Artificial Intelligence 172, no. 1 (January): 1-18.

42. Kraus, Sarit, Katia Sycara, and Amir Evenchik. 1998. Reaching agreements through argumentation: a logical model and implementation. Artificial Intelligence 104, no. 1-2 (September): $1-69$.

43. Kraus, Sarit, Jonathan Wilkenfeld, and Gilad Zlotkin. 1995. Multiagent negotiation under time constraints. Artificial Intelligence 75, no. 2 (June): 297-345.

44. Lai, Guoming, Li Cuihong, Katia P. Sycara, and Joseph Giampapa. 2004. Literature Review on Multi-attribute Negotiations. December 6.

45. Lai, Guoming, Cuihong Li, and Katia Sycara. 2006. Efficient Multi-Attribute Negotiation with Incomplete Information. Group Decision and Negotiation 15, no. 5: 511-528.

46. Lai, Guoming, and Katia Sycara. 2009. A Generic Framework for Automated Multi-attribute Negotiation. Group Decision and Negotiation 18, no. 2 (March 1): 169-187.

47. Lai, Guoming, Katia Sycara, and Cuihong Li. 2008. A decentralized model for automated multi-attribute negotiations with incomplete information and general utility functions. Multiagent and Grid Systems 4, no. 1 (January 1): 45-65.

48. Lambert, Lynn, and Sandra Carberry. 1992. Modeling negotiation subdialogues. In Proceedings of the 30th annual meeting on Association for Computational Linguistics, 193-200. Newark, Delaware: Association for Computational Linguistics.

49. Lang, Kevin, and Robert W. Rosenthal. 2001. Bargaining Piecemeal Or All At Once? The Economic Journal 111, no. 473: 526-540.

50. Lewicki, Roy J., Stephen E. Weiss, and David Lewin. 1992. Models of Conflict, Negotiation and Third Party Intervention: A Review and Synthesis. Journal of Organizational Behavior 13, no. 3 (May): 209-252.

51. Li, Cuihong, Joseph Giampapa, and Katia P. Sycara. 2006. Bilateral negotiation decisions with uncertain dynamic outside options. Systems, Man, and Cybernetics, Part C: Applications and Reviews, IEEE Transactions on 36, no. 1: 31-44.

52. Li, Cuihong, and Gerald Tesauro. 2003. A strategic decision model for multi-attribute bilateral negotiation with alternating. In Proceedings of the 4th ACM conference on Electronic commerce, 208-209. San Diego, CA, USA: ACM.

53. Licklider, Roy. 1995. The Consequences of Negotiated Settlements in Civil Wars, 1945-1993. The American Political Science Review 89, no. 3 (September): 681-690.

54. Lin, Raz, Sarit Kraus, Jonathan Wilkenfeld, and James Barry. 2008. Negotiating with bounded rational agents in environments with incomplete information using an automated agent. Artificial Intelligence 172, no. 6-7 (April): 823-851.

55. Lochbaum, Karen E. 1998. A collaborative planning model of intentional structure. Comput. Linguist. 24, no. 4: 525-572.

56. Luce, Duncan, and Howard Raiffa. 1989. Games and Decisions : Introduction and Critical Survey. Dover Publications, April 1.

57. Luo, Xudong, Nicholas R. Jennings, Nigel Shadbolt, Ho-fung Leung, and Jimmy Ho-man Lee. 2003. A fuzzy constraint based model for bilateral, multi-issue negotiations in semicompetitive. Artificial Intelligence 148: 53-102. 
58. McGinn, Kathleen L., and Angela T. Keros. 2002. Improvisation and the Logic of Exchange in Socially Embedded Transactions. Administrative Science Quarterly 47, no. 3 (September): 442-473.

59. Morre, Don. 2002. The unexpected benefits of final deadlines in negotiation. Journal of Experimental Social Psychology 40, no. 1: 121-127.

60. Mumpower, Jeryl L. 1991. The Judgment Policies of Negotiators and the Structure of Negotiation Problems. Management Science 37, no. 10 (October): 1304-1324. doi:10.2307/2632402.

61. Muthoo, Abhinay. 1995. On the Strategic Role of outside Options in Bilateral Bargaining. Operations Research 43, no. 2 (April): 292-297. doi:10.2307/171837.

62. Nash, John. 1951. Non-Cooperative Games. The Annals of Mathematics 54, no. 2: 295, 286.

63. Nash, John. 1953. Two-Person Cooperative Games. Econometrica 21, no. 1: 140, 128.

64. Neale, Margaret A., and Max H. Bazerman. 1985. The Effects of Framing and Negotiator Overconfidence on Bargaining Behaviors and Outcomes. The Academy of Management Journal 28, no. 1 (March): 34-49.

65. Neelin, Janet, Hugo Sonnenschein, and Matthew Spiegel. 1988. A Further Test of Noncooperative Bargaining Theory: Comment. The American Economic Review 78, no. 4 (September): 824-836.

66. Netto, Gina. 2008. Multiculturalism in the Devolved Context: Minority Ethnic Negotiation of Identity through Engagement in the Arts in Scotland. Sociology 42, no. 1 (February 1): 47-64.

67. Ochs, Jack, and Alvin E. Roth. 1989. An Experimental Study of Sequential Bargaining. The American Economic Review 79, no. 3 (June): 355-384.

68. Parsons, Simon, and N. R Jennings. 1996. Negotiation Through Argumentation-a Preliminary Report.

69. Paruchuri, Praveen, Nilanjan Chakraborty, Roie Zivan, Katia Sycara, Miroslav Dudik, and Geoff Gordon. 2009. POMDP based Negotiation Modeling. In proceedings of the first MICON (Modeling Intercultural Collaboration and Negotiation) workshop, 66-78. Pasedena, CA, July 13.

70. Ponsati, Clara, and Joel Watson. 1997. Multiple-issue bargaining and axiomatic solutions. International Journal of Game Theory 26, no. 4 (December 1): 501-524.

71. Raith, Matthias G. 2000. Fair-negotiation procedures. Text.

72. Rangaswamy, Arvind, and G. Richard Shell. 1997. Using Computers to Realize Joint Gains in Negotiations: Toward an "Electronic Bargaining Table". Management Science 43, no. 8 (August): 1147-1163.

73. Rhoades, Gary, and Sheila Slaughter. 1991. Professors, Administrators, and Patents: The Negotiation of Technology Transfer. Sociology of Education 64, no. 2 (April): 65-77.

74. Robu, Valentin, D. J. A. Somefun, and J. A. La Poutr. 2005. Modeling complex multi-issue negotiations using utility graphs. In Proceedings of the fourth international joint conference on Autonomous agents and multiagent systems, 280-287. The Netherlands: ACM.

75. Roth, Alvin E. 1985. Game-theoretic models of bargaining. Cambridge University Press.

76. Roth, Alvin E., and M.W.K. Malouf. 1979. Game-theoretic models and the role of information in bargaining. Psychological review 86: 574-594.

77. Roth, Alvin E., Michael W. K. Malouf, and J. Keith Murnighan. 1981. Sociological versus strategic factors in bargaining. Journal of Economic Behavior \& Organization 2, no. 2 (June): 153-177.

78. Roth, Alvin E., and J. Keith Murnighan. 1982. The Role of Information in Bargaining: An Experimental Study. Econometrica 50, no. 5 (September): 1123-1142.

79. Roth, Alvin E., and Francoise Schoumaker. 1983. Subjective Probability and the Theory of Games: Some Further Comments. Management Science 29, no. 11 (November): 1337-1340.

80. Rothkopf, Michael H. 1983. Modeling Semirational Competitive Behavior. Management Science 29, no. 11 (November): 1341-1345.

81. Sebenius, James K. 1992. Negotiation Analysis: A Characterization and Review. Management Science 38, no. 1 (January): 18-38. 
82. Shakun, Melvin F. 1991. Airline Buyout: Evolutionary Systems Design and Problem Restructuring in Group Decision and Negotiation. Management Science 37, no. 10 (October): 1291-1303.

83. Shubik, Martin. 1983. Comment on "The Confusion of Is and Ought in Game Theoretic Contexts". Management Science 29, no. 12 (December): 1380-1383.

84. Snyder, C R, and R L Higgins. 1988. Excuses: their effective role in the negotiation of reality. Psychological Bulletin 104, no. 1 (July): 23-35.

85. Sycara, Katia P. 1989. Argumentation: Planning Other Agents' Plans. In Proceedings of the Eleventh International Joint Conference on Artificial Intelligence.

86. Sycara, Katia P. 1990a. Negotiation planning: An AI approach. European Journal of Operational Research 46, no. 2 (May): 216-234.

87. Sycara, Katia P. 1990b. Persuasive argumentation in negotiation. Theory and Decision 28, no. 3 (May 1): 203-242.

88. Sycara, Katia P. 1991. Problem Restructuring in Negotiation. Management Science 37, no. 10 (October): 1248-1268.

89. Thompson, Leigh L. 1991. Information exchange in negotiation. Journal of Experimental Social Psychology 27, no. 2: 161-179.

90. Thompson, Leigh L. 1996. Lost-Lose Agreement in Interdependent Decision Making. Psychological bulletin 120, no. 3: 396-409.

91. Tohm, Fernando. 2002. Negotiation and Defeasible Decision Making. Theory and Decision 53, no. 4 (December 1): 289-311.

92. Ulvila, Jacob W., and Warren D. Snider. 1980. Negotiation of International Oil Tanker Standards: An Application of Multiattribute Value Theory. Operations Research 28, no. 1 (February): 81-96.

93. Weingart, L.R., R.J. Bennett, and Jeanne M. Brett. 1993. The impact of consideration of issues and motivational orientation on group negotiation process and outcome. Journal of Applied Psychology 78,.

94. Zartman, I. William, and Maureen R. Berman. 1983. The Practical Negotiator. Yale University Press, September 10.

95. Zeng, Dajun, and Katia Sycara. 1998. Bayesian learning in negotiation. Int. J. Hum.-Comput. Stud. 48, no. 1: 125-141.

96. Zlotkin, Gilad, and Jeffrey S. Rosenschein. 1996. Mechanism design for automated negotiation, and its application to task oriented domains. Artificial Intelligence 86, no. 2 (October): 195-244. 